\title{
Continuous melting of a partially pinned two-dimensional vortex lattice in a square array of pinning centers
}

\author{
Toby Joseph* and Chandan Dasgupta ${ }^{\dagger}$ \\ Centre for Condensed Matter Theory, Department of Physics, Indian Institute of Science, Bangalore 560 012, India
}

\begin{abstract}
The structure and equilibrium properties of a two-dimensional system of superconducting vortices in a periodic pinning potential with square symmetry are studied numerically. For a range of the strength of the pinning potential, the low-temperature crystalline state exhibits only one of the two basic periodicities (in the $x$ and $y$ directions) of the pinning potential. This "partially pinned" solid undergoes a continuous melting transition to a weakly modulated liquid as the temperature is increased. A spin model, constructed using symmetry arguments, is shown to reproduce the critical behavior at this transition.
\end{abstract}

Thin-film superconductors with artificially constructed periodic arrays of pinning centers have attracted much experimental $^{1-3}$ and theoretical ${ }^{4}$ attention in recent years. Such arrays may consist of microholes ("antidots"), ${ }^{1}$ defects produced by heavy-ion bombardment, ${ }^{2}$ or magnetic dots. ${ }^{3}$ The presence of a periodic pinning potential has many interesting effects on the equilibrium and transport properties of a system of vortices induced in the sample by an external magnetic field. Some of these effects, which depend crucially on the value of the filling factor $n$ that measures the average number of vortices per unit cell of the pin lattice, have been observed in imaging experiments ${ }^{2}$ and in measurements of the magnetic ${ }^{1}$ and transport $^{3}$ properties of such samples. The melting transition of the vortex lattice in such systems provides an example of two-dimensional $(2 d)$ melting in an external periodic potential. Evidence for this melting transition has been found in imaging experiments ${ }^{2}$ and magnetization measurements. ${ }^{1}$ Similar melting transitions are of interest in other physical systems such as atoms adsorbed on crystalline substrates, ${ }^{5}$ arrays of Josephson junctions, ${ }^{6}$ and colloidal particles in interfering laser fields. ${ }^{7}$

The effects of a weak, commensurate, periodic potential on $2 d$ melting have been studied ${ }^{5}$ within the framework of the Kosterlitz-Thouless-Halperin-Nelson-Young theory ${ }^{5,8}$ of defect mediated melting. For $n \ll 1$, this analysis predicts the occurrence of two continuous transitions: a depinning transition from a low-temperature pinned solid phase in registry with the substrate to a floating solid phase that is essentially decoupled from the substrate, and a melting transition at a higher temperature where the floating solid transforms to a liquid. This sequence of transitions has been observed in simulations ${ }^{4,6}$ of $2 d$ systems in weak, commensurate, periodic potentials with triangular and square symmetry. The two transitions are expected to merge into a single one (from the pinned solid to the liquid) as $n$ is increased ${ }^{5,6}$ and/or the pinning potential is strengthened. ${ }^{4}$

In this paper, we report the results of Monte Carlo (MC) simulations of the equilibrium properties of a $2 d$ system of vortices in the presence of a square array of pinning centers. The filling factor $n$ is taken to be unity. We consider pinning centers that produce a repulsive potential with a range comparable to that of the intervortex interaction. Pinning centers with these properties may be experimentally realized in arrays of magnetic dots each of which produces a potential that can be tailored ${ }^{9}$ by adjusting its magnetic moment. Another physical realization is obtained in a square array of strong, attractive, short-range pinning centers at filling $n=2 .^{4}$ In this case, each pinning center would trap a vortex at low temperatures, and these pinned vortices would interact with the remaining interstitial vortices (assuming each pin can trap only one vortex) via an effective repulsive potential. ${ }^{10}$ The net potential produced by an array of such pinning centers has very flat minima ${ }^{10}$ at the centers of the square unit cells of the pin lattice (see Fig. 1). This kind of pinning is qualitatively different from that considered in previous studies ${ }^{4,6}$ in which each pinning center was assumed to produce an attractive potential with range much smaller than the intervortex spacing.

We find that this difference in the nature of the pinning has strong effects on the structure of the low-temperature solid phase and its melting transition. For a range of values

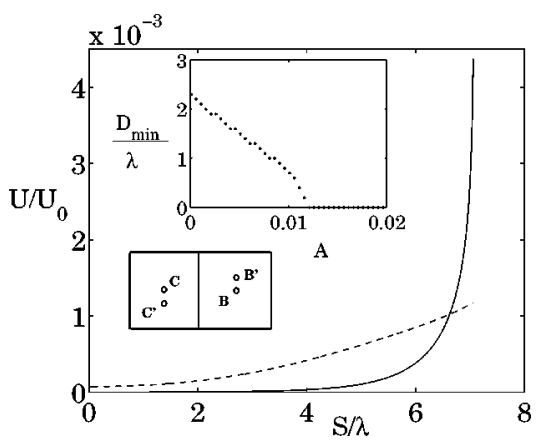

FIG. 1. The main plot shows the net pinning potential for $A$ $=10^{-3}$ (solid line) and the potential due to vortices located at the centers of the squares (dashed line) as functions of the distance $S$ from the center of a pin square along a diagonal. The upper inset shows a plot of $D_{\min }$, the displacement from the center that minimizes the lattice energy, as a function of the pinning-strength parameter $A$. The lower inset shows an unit cell of the partially pinned structure. $B$ and $C$ are the positions of the vortices in the state with square symmetry and $B^{\prime}$ and $C^{\prime}$ are their positions in the partially pinned structure, with $B B^{\prime}=C C^{\prime}=D_{\text {min }}$. Three other structures, related to the shown one by symmetry, are equally probable at low temperatures. 
of the strength of the pinning potential, the low-temperature state of our vortex system is a lattice with a basis, with unit cells of size $2 d \times d$, where $d$ is the spacing of the pin lattice, and two vortices in each unit cell (see Fig. 1). Three other structures, related by symmetry to the one shown in Fig. 1, are equally likely to occur at low temperatures. We call this phase, which exhibits one of the two basic periodicities of the square pin array, a partially pinned solid to distinguish it from the pinned and floating solid phases mentioned above.

As the temperature is increased, the partially pinned solid undergoes a continuous melting transition to a weakly modulated liquid that has the square symmetry of the substrate. We have used finite-size scaling to analyze the critical behavior at this transition. We have also used symmetry considerations to construct a spin model that is expected to exhibit a transition in the same universality class as that of the melting transition in the vortex system. The values of critical exponents obtained from a finite-size scaling analysis of the results of simulations of the spin model are consistent with those obtained for the vortex system. This transition appears to belong in a universality class not found in previous studies of similar systems.

We model the $2 d$ system of vortices as a collection of point particles interacting via the repulsive potential

$$
U(r)=U_{0} K_{0}(r / \lambda)
$$

Here, $K_{0}$ is the Hankel function and $U_{0}=\Phi_{0}^{2} t /\left(8 \pi^{2} \lambda^{2}\right)$, where $\Phi_{0}$ is the flux quantum, $\lambda$ is the penetration depth, and $t$ is the film thickness. The interaction of the vortices with the pinning centers is assumed to be of the form $A U(r)$, where the parameter $A$ measures the relative strength of the pinning potential. We use parameters appropriate for the $\mathrm{Nb}$ sample studied in Ref. 2: $\lambda=0.1 \mu \mathrm{m}, d=10 \lambda, t=\lambda$. For these parameter values, $U(r=d) / k_{B} \simeq 7 \mathrm{~K}$. We measure lengths in units of $\lambda$, energies in units of $U_{0}$, and the temperature in Kelvins.

In Fig. 1, we have shown the variation of the net pinning potential for $A=10^{-3}$ along a diagonal of a pin square, and compared it with the potential due to a system of vortices located at the centers of the pin squares. The pining potential exhibits a very flat minimum at the center of the square. This plot also shows that the pinning and interaction energies are comparable for this value of $A$. We used a simulated annealing procedure to find the ground states of the vortex system for various values of $A$. When $A$ is of order unity or higher, the ground state has square symmetry, with one vortex located at the center of each elementary square of the pin array. As $A$ is reduced below $A_{c} \simeq 0.012$, the ground state is found to be made up of unit cells that consist of two pin squares. The vortices in the two squares are displaced from the centers by equal amounts in opposite directions, as shown in the lower inset of Fig. 1. This displacement causes a reduction of the interaction energy, which more than compensates the increase in the pinning energy if $A$ is small. The distance $D_{\min }$ of the vortices from the center of the square increases as $A$ is decreased, and reaches a maximum of about $0.23 d$ for $A$ $\approx 10^{-4}$. We have also calculated the energy of a vortex lattice of this structure for different values of the displacement

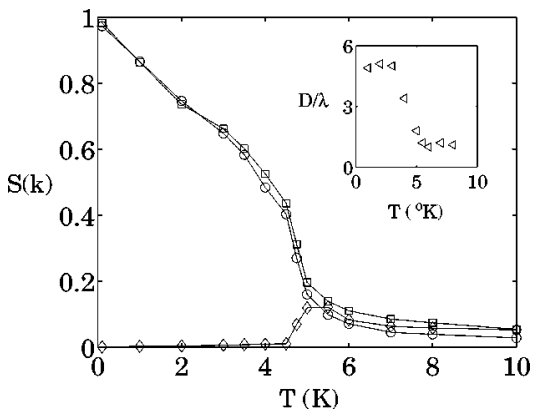

FIG. 2. Temperature dependence of the structure functions $S(\mathbf{G})$ for $A=10^{-4}$. Data for three values of $\mathbf{G}$ (see text), $\mathbf{G}_{1}$ (squares), $\mathbf{G}_{2}$ (circles), and $\mathbf{G}_{3}$ (diamonds) are shown. The solid lines are guides to the eye. The inset shows the average displacement $D$ of the vortices from the centers of the pin squares as a function of the temperature $T$.

$D$ from the center and found the $D$ that minimizes the energy for different $A$. The results, shown in Fig. 1, match well with those obtained from simulated annealing. These results clearly show a transition from a fully pinned structure with square symmetry to a partially pinned structure with $D_{\min }$ $\neq 0$ as $A$ is decreased below $A_{c}$. The ground states for $A$ $<10^{-5}$ appear to have a complex, disordered structure (we are not sure that our simulated annealing procedure located the true ground states for such values of $A$ ), and the triangular Abrikosov lattice is recovered for $A=0$.

At high temperatures, the vortices form a weakly modulated liquid with square symmetry. We used MC simulations to study how the system evolves to this state as the temperature $T$ is increased. We monitored structural changes by measuring the structure functions $S(\mathbf{k})=\langle\rho(\mathbf{k}) \rho(-\mathbf{k})\rangle / N^{2}$, where $\langle\cdots\rangle$ represents a thermodynamic (MC) average, $\rho(\mathbf{k})$ is the Fourier transform of the local density, and $N$ is the number of particles in the system. We also looked for signatures of a transition by measuring the specific heat, $C_{v}=\left\langle(E-\langle E\rangle)^{2}\right\rangle /\left(N k_{B} T^{2}\right)$, where $E$ is the total energy of the system. When $A$ is large enough to have a lattice with square symmetry as the ground state, the system gradually transforms to a modulated liquid with the same symmetry with no signature of a phase transition. For lower values of $A$, when the symmetry of the ground state is different from that of the high-temperature phase, the system undergoes a continuous transition that is signalled by a peak in the specific heat and sharp changes in $S(\mathbf{k})$ for appropriate k's, as shown in Figs. 2 and 3.

The reciprocal lattice vectors of the structure with the unit cell shown in Fig. 1 are $\mathbf{G}\left(n_{1}, n_{2}\right)=\left[n_{1}(\pi / d), n_{2}(2 \pi / d)\right]$, where $n_{1}$ and $n_{2}$ are integers. For this structure, $\langle\rho(\mathbf{G})\rangle$ vanishes for the smallest $\mathbf{G}$ corresponding to $n_{1}=1, n_{2}=0$. We have measured the temperature-dependence of $S(\mathbf{G})$ for the next three smallest $\mathbf{G s :} \mathbf{G}_{1}=\mathbf{G}(2,0), \mathbf{G}_{2}=\mathbf{G}(1,1)$, and $\mathbf{G}_{3}$ $=\mathbf{G}(0,1)$. Note that $\mathbf{G}_{1}$ and $\mathbf{G}_{3}$ are reciprocal lattice vectors of the square pin array, but $\mathbf{G}_{2}$ is not. Simulation data obtained in a cooling run for a sample with $A=10^{-4}$ are shown in Fig. 2. At high temperatures, $S\left(\mathbf{G}_{1}\right)$ and $S\left(\mathbf{G}_{3}\right)$ have the same small value, whereas $S\left(\mathbf{G}_{2}\right)$ is smaller, indicating a 


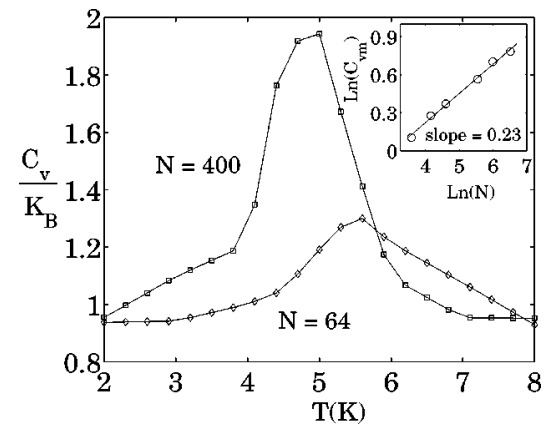

FIG. 3. The specific heat $C_{v}$ (in units of $k_{B}$ ) as a function of the temperature $T$ for $A=10^{-4}$ and two sample sizes, $N=64$ and $N$ $=400$. The lines joining the data points are guides to the eye. The inset shows a double-log plot of $C_{v m}$, the peak value of the specific heat, as a function of the system size $N=L^{2}$.

weakly modulated phase with square symmetry. As the temperature is decreased, $S\left(\mathbf{G}_{1}\right)$ and $S\left(\mathbf{G}_{2}\right)$ increase sharply near $T \simeq 5.0 \mathrm{~K}$, while $S\left(\mathbf{G}_{3}\right)$ decreases at about the same temperature. As the temperature is decreased further, the values of $S\left(\mathbf{G}_{1}\right)$ and $S\left(\mathbf{G}_{2}\right)$ approach unity, while $S\left(\mathbf{G}_{3}\right)$ goes to zero, indicating a partially pinned structure. As shown in the inset of Fig. 2, the average displacement $D$ of the vortices from the centers of the pin squares exhibits a sharp increase as the temperature is decreased across $T \simeq 5.0 \mathrm{~K}$. These results strongly suggest a phase transition between the partially pinned state and a state with square symmetry at $T=T_{c}$ $\simeq 5 \mathrm{~K}$.

In order to determine the nature of the transition, we have carried out a finite-size scaling study of the specific heat $C_{v}$ of the system. As shown in Fig. 3, $C_{v}$ peaks at the transition temperature obtained from the behavior of $S(\mathbf{G})$ and $D$, and the peak becomes higher and sharper as the size of the system is increased. This is the behavior expected at a continuous phase transition for which finite-size scaling theory ${ }^{11}$ predicts that the peak value of $C_{v}$ should scale as $L^{\alpha / \nu}$, where $L=\sqrt{N}$ is the linear size of the system, and $\alpha$ and $\nu$ are, respectively, the critical exponents for the specific heat and the correlation length. As shown in the inset of Fig. 3, our data are quite consistent with this behavior, with $\alpha / \nu$ $\simeq 0.46$. Moreover, we have checked for hysteresis by measuring the specific heat during heating and cooling runs. We did not find any evidence for hysteresis, confirming that the transition is a continuous one. The transition temperature is found $^{12}$ to depend nonmonotonically on the strength $A$ of the pinning potential. As $A$ is decreased below a critical value near $10^{-2}$, the transition temperature increases from zero, attains a maximum near $A=8 \times 10^{-5}$, and then decreases as $A$ is decreased further.

We have used symmetry arguments to construct a spin model that should exhibit a phase transition in the same universality class as the transition found in the vortex system. As noted earlier, the vortex system has four degenerate ground states: two in which the displacements from the centers of the pin squares are $\pm D_{\min } \hat{\mathbf{y}}\left(\mp D_{\min } \hat{\mathbf{y}}\right)$ in even (odd) columns of the pin lattice, and two others in which the displacements are $\pm D_{\min } \hat{\mathbf{x}}\left(\mp D_{\min } \hat{\mathbf{x}}\right)$ in even (odd) rows $(\hat{\mathbf{x}}$ and $\hat{\mathbf{y}}$ are unit vectors in the horizontal and vertical directions, respectively). We, therefore, consider four-state planar "spin" variables $\boldsymbol{\sigma}_{i}$ located at the sites of the dual of the pin lattice. Each of these variables has unit length and can point in the four directions, $\pm \hat{\mathbf{x}}$, and $\pm \hat{\mathbf{y}}$. One may think of these variables as representing the directions of small displacements of magnitude $\delta$ from the centers of the pin squares. The distance $s_{i j}$ between two vortices in neighboring pin squares, with displacements $\delta \boldsymbol{\sigma}_{i}$ and $\delta \boldsymbol{\sigma}_{j}$, is given by

$$
s_{i j}^{2}=d^{2}+2 \delta^{2}\left(1-\boldsymbol{\sigma}_{i} \cdot \boldsymbol{\sigma}_{j}\right)+2 \delta d\left(\sigma_{j \alpha}-\sigma_{i \alpha}\right),
$$

where $\alpha$ is $x(y)$ if the spins $\boldsymbol{\sigma}_{i}$ and $\boldsymbol{\sigma}_{j}$ are separated horizontally (vertically). Since the intervortex interaction depends only on the distance, and the pinning potential is independent of the orientation of the $\boldsymbol{\sigma}$ 's, the symmetry of the vortex problem would be preserved in the spin model if its Hamiltonian is taken to be a suitably chosen function of $s^{2}$ that leads to the fourfold-degenerate ground-state structures described above. We have found that the Hamiltonian

$$
\mathcal{H}=J_{o} \sum_{\langle i j\rangle} \exp \left(-s_{i j}^{2} / s_{0}^{2}\right),
$$

where the sum is over nearest-neighbor pairs and $J_{0}$ is an energy parameter, leads to the expected ground-state structure if the length parameter $s_{0}$ is sufficiently large. By expanding the exponential in Eq. (3) and making use of the properties of the $\boldsymbol{\sigma}_{i}$ 's, this Hamiltonian may be written as

$$
\begin{aligned}
\mathcal{H}= & \sum_{\langle i j\rangle}\left[J_{1} \sigma_{i \alpha} \sigma_{j \alpha}+J_{2} \sigma_{i \beta} \sigma_{j \beta}+J_{3} \sigma_{i \alpha} \sigma_{j \alpha}\left(\sigma_{i \alpha}-\sigma_{j \alpha}\right)\right. \\
& \left.+J_{4} \sigma_{i \alpha}^{2} \sigma_{j \alpha}^{2}+J_{5} \sigma_{i \beta}^{2} \sigma_{j \beta}^{2}\right]
\end{aligned}
$$

where $\alpha, \beta$ are $x, y(y, x)$ for horizontal (vertical) bonds, and $J_{1}, J_{2}, J_{3}, J_{4}$, and $J_{5}$ are functions of $J_{0}, \delta, d$, and $s_{0}$. While the spins in our model are analogous to those in the fourstate clock model, the Hamiltonian of Eq. (4) does not have the $z(4)$ symmetry of the clock model.

We have performed extensive MC simulations of the thermodynamics of the spin model for $J_{0}=10$ (this sets the temperature scale), $d / s_{0}=2$ and $d / \delta=4$. For these parameter values, we find four degenerate ground states in which the spins point in $\pm \hat{\mathbf{x}}$ directions in alternate rows, or in $\pm \hat{\mathbf{y}}$ directions in alternate columns. The order-parameter $m$ is defined as

$$
m=\frac{1}{N}\left\langle\left|\Sigma_{1} \sigma_{i x}-\Sigma_{2} \sigma_{i x}\right|+\left|\Sigma_{3} \sigma_{i y}-\Sigma_{4} \sigma_{i y}\right|\right\rangle,
$$

where the four sums $\Sigma_{k}, k=1-4$ are over even rows, odd rows, even columns, and odd columns, respectively. This definition ensures that $m=1$ in any of the four ground states. The transition temperature $T_{c}$ and the correlation-length exponent $\nu$ were determined from a finite-size scaling analysis of the data for the Binder cumulant ${ }^{11}$ for the order parameter, $c_{m} \equiv 1-\left\langle m^{4}\right\rangle /\left(3\left\langle m^{2}\right\rangle^{2}\right)$. The $L$ and $T$ dependence of this quantity near the transition is expected to have the form $c_{m}(L, T)=f\left(L^{1 / v} t\right)$, where $f$ is a scaling function and $t=(T$ 


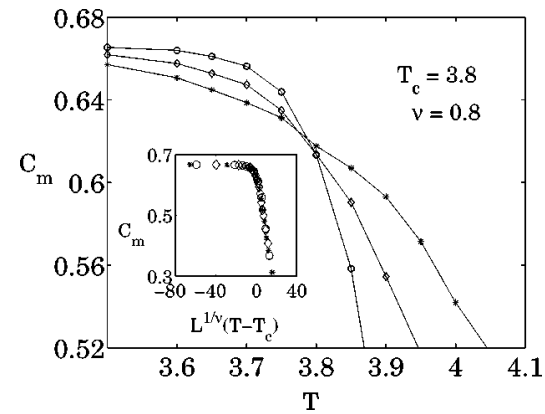

FIG. 4. The main plot shows the order parameter cumulant $c_{m}$ of the spin model (see text) as a function of temperature $T$ for three system sizes, $N=144$ (stars), $N=324$ (diamonds), and $N=1024$ (circles). The inset shows a finite-size scaling plot of $c_{m}$ versus $L^{1 / \nu}\left(T-T_{c}\right)$ with $T_{c}=3.8$ and $\nu=0.8$.

$\left.-T_{c}\right) / T_{c}$. As shown in Fig. 4, plots of $c_{m}$ vs $T$ for different sample sizes intersect at $T=T_{c} \simeq 3.8$, confirming the occurrence of a continuous transition. The inset of Fig. 4 shows that the data for $c_{m}$ for different $L$ and $T$ collapse to the same scaling curve when plotted against $L^{1 / \nu}\left(T-T_{c}\right)$ with $\nu$ $=0.8$. The other critical exponents, computed from finitesize scaling analysis of specific heat, order-parameter, and susceptibility data ${ }^{12}$ are $\beta \simeq 0.05, \gamma \simeq 1.5$, and $\alpha \simeq 0.4$. These values are consistent, within error bars, with the value of $\alpha / \nu$ obtained for the vortex system. These exponent values establish that the transition in our model is not in the universality class of the four-state clock model. The observed critical behavior is also quite different from that expected ${ }^{5,8}$ near a Kosterlitz-Thouless-type transition. It appears that the universality class of this transition is different from those found in earlier studies of similar systems.

To conclude, we have shown that a $2 d$ system of particles in a periodic potential with square symmetry can have a partially pinned low-temperature phase that undergoes a continuous transition to a weakly modulated liquid as the temperature is increased. After the submission of this paper, we came across two recent papers that confirm some of our predictions. An experimental study ${ }^{13}$ has analyzed the vortex structure at the bottom surface of a thin superconductor with a commensurate square array of pinning centers on the top surface. Vortex lines pinned at the top surface provide, via their elastic energy, an effective pinning potential of square symmetry at the bottom surface whose strength is a decreasing function of the sample thickness. The experiment finds a structure with the same symmetry as that of our "partially pinned" lattice for a range of thicknesses. A numerical modeling ${ }^{14}$ of the experimental system also yields results quite similar to those shown in the insets of Fig. 1.
*Electronic address: toby@physics.iisc.ernet.in

†Electronic address: cdgupta@physics.iisc.ernet.in

${ }^{1}$ M. Baert, V.V. Metlushko, R. Jonckheere, V.V. Moshchalkov, and Y. Bruynseraede, Phys. Rev. Lett. 74, 3269 (1995).

${ }^{2}$ K. Harada, O. Kamimura, H. Kasai, T. Matsuda, A. Tonomura, and V.V. Moshchalkov, Science 274, 1167 (1996).

${ }^{3}$ J.I. Martin, M. Vélez, J. Nogués, and Ivan K. Schuller, Phys. Rev. Lett. 79, 1929 (1997).

${ }^{4}$ C. Reichhardt, C.J. Olson, R.T. Scalettar, and G.T. Zimányi, Phys. Rev. B 64, 144509 (2001), and references therein.

${ }^{5}$ D.R. Nelson and B.I. Halperin, Phys. Rev. B 19, 2457 (1979).

${ }^{6}$ M. Franz and S. Teitel, Phys. Rev. Lett. 73, 480 (1994); Phys. Rev. B 51, 6551 (1995).

${ }^{7}$ Q.-H. Wei, C. Bechinger, D. Rudhardt, and P. Leiderer, Phys. Rev.
Lett. 81, 2606 (1998).

${ }^{8}$ J.M. Kosterlitz and D.J. Thouless, J. Phys. C 6, 1181 (1973); A.P. Young, Phys. Rev. B 19, 1855 (1979).

${ }^{9}$ I.F. Lyuksyutov and V. Pokrovsky, Phys. Rev. Lett. 81, 2344 (1998).

${ }^{10}$ I.B. Khalfin and B.Ya. Shapiro, Physica C 207, 359 (1993).

${ }^{11}$ Applications of the Monte Carlo Method in Statistical Physics, edited by K. Binder (Springer, Berlin, 1984).

${ }^{12}$ T. Joseph and C. Dasgupta, (unpublished).

${ }^{13}$ Y. Fasano, M. De Seta, M. Menghini, H. Pastoriza, and F. de la Cruz, cond-mat/0208537 (unpublished).

${ }^{14}$ P.S. Cornaglia and M.F. Laguna, cond-mat/0209565 (unpublished). 